\title{
A Method Based Genetic Algorithm for Pipe Routing Design
}

\author{
Changtao Wang ${ }^{1,}$, , Xiaotong Sun ${ }^{2, b}$,Tiancheng Yuan ${ }^{3, c}$ \\ ${ }^{1}$ School of Information and Control Engineering,Shenyang Jianzhu University,Shenyang \\ China, 110168 \\ ${ }^{2}$ School of Information and Control Engineering,Shenyang Jianzhu University,Shenyang \\ China, 110168 \\ ${ }^{3}$ School of Information and Control Engineering,Shenyang Jianzhu University, Shenyang \\ China, 110168 \\ a542736170@qq.com, b18809821962@163.com, ${ }^{\circ} 526697112 @ q q . c o m$
}

\begin{abstract}
Keywords: pipe routing design, 3-dimensional space, distance constraint, genetic algorithm Abstract. This paper discusses a pipe routing design method which uses genetic algorithm (GA) to find the feasible and suitable paths in an environment with some certain constraints, especially distance constraint. The algorithm uses a grid processing method first to present 3-dimensional building environment. An effective evaluation method is specially developed for the proposed genetic algorithm. The evaluation method is able to avoid obstacles, reduce the number of bends and satisfy the distance need. The proposed GA uses problem-specific genetic algorithms for pipe routing design instead of standard GA. The proposed method based genetic algorithm incorporates the domain knowledge of pipe routing design into its specialized operators.
\end{abstract}

\section{Introduction}

In a building, facilities provide a convenient and comfortable living environment for people, pipes connected to the facilities are inseparable. Layout of the building pipelines are very complex, often causing confusion or waste of building space. If the pipeline comprehensive arrangement is not carried out in advance at the design stage of, it will bring a lot of inconvenience to the installation as well as building construction, even affect the construction quality and safety. Therefore, a comprehensive arrangement of pipeline is very important [1]. In recent years, BIM technology, was introduced to our country and has been highly valued. BIM change construction industry, information technology will fundamentally backwardness. But in terms of its entire development process, BIM technology still immature. As a result, it's necessary to combine BIM technology and intelligent algorithms together to achieve the automatic layout of pipeline.

Pipe routing design is an important issue in the process of building design. In an environment with facilities and other pipes, the aim of pipe routing design is to find a suitable collision-free path for pipes. Very often this path is highly desirable to be optimal or near-optimal with respect to path length, bends' number and layout near or far away from something. These factors are commonly adopted design criteria. In recent years, pipe routing design has been an active research area, and many methods have been developed to solve this problem, such as ant colony algorithm, particle swarm optimization algorithm and maze algorithm. Each method has its own strength over others in certain aspects. Researches have always been seeking alternative and more efficient ways to solve the problem.

There is no doubt that path routing design can be viewed as an optimization problem under certain constraints. Many researchers have applied GA to path plan for pipe routing design .However, most of those methods use nearly the same evaluation, and a few modifications were made to the genetic operators.

In terms of the study of pipe routing design, it is mainly focus on the single pipe routing design. However, the layout for the facilities and other pipes' location may also affect the pipe routing design. Some approaches for pipe routing design are able to cope with obstacles .In [2], a pipe routing design based on GA was proposed. This approach utilize a special code for GA, the 3-dimensional space is pre-processing by grids to produce many points, and give 3-dimensional coordinates to each point. 
Naturally the pipeline is represent by the coordinates. With this special code, the GA's operators are modified correspondingly. The workers working space are considered when the pipeline designed. It combines fuzzy algorithm and genetic algorithm. In [3], based on ant colony algorithm for multi-pipe routing design, the method needs to evaluate partial and global paths optimal by two fitness functions.

However, few of the study concentrate on the distance constraint for each pipe routing design. In this paper, a method based genetic algorithm for pipe routing design is proposed to solve distance constraint for the pipe. The proposed GA uses problem-specific genetic algorithm for pipe routing design. Also, the algorithm uses a grid processing method to present 3-dimensional building environment with obstacles and facilities. In accord with the pipe distance constraint problem, an effective evaluation method is especially developed for the proposed genetic algorithm. Two evaluation functions are added to the evaluation to evaluate the distance constraint. The proposed method based genetic algorithm incorporates the domain knowledge into its specialized operators[4].

\section{Organization of the Text}

The proposed genetic algorithm feature is its effective evaluation method and its special genetic operators.

\section{Problem Representation}

Representation is a key issue in the work of GA. The proposed genetic algorithm uses grids processing to get points in 3-dimensional space. A potential pipeline is formed by several points, from the start point $S$ to the target point $T$. Grids function in this representation is to discretize the whole environment and also helps the pipe keep straight, not lean.

\section{Evaluation}

The aim of proposed method based GA is to optimal the pipe routing design. As a result, the evaluation should be able to distinguish the qualities of feasible pipeline. The feasible pipeline is formed in a special way, using coordinate of points. Having considered common pipe design criteria, such as short length, few bends and layout close to or far away from something, the pipe distance constraints are viewed as an important role in this paper. In order to solve pipe distance constraints problem, two functions are proposed- $S$ and $O$.

Here are an example to demonstrate the pipe distance constraints. There are three pipes, Pipe a, Pipe $\mathrm{b}$ and Pipe $\mathrm{c}$. The design rules of these three pipes are Pipe a should be designed $3 \mathrm{~cm}$ away from the wall; Pipe $\mathrm{b}$ should be designed $10 \mathrm{~cm}$ away from the wall and its location is above Pipe a when a, $\mathrm{b}$ designed vertical or right-side of Pipe a when a, b designed parallel; Pipe c should be designed far away from Pipe a $30 \mathrm{~cm}$.From above rules, three basic problems are put forward. Problem 1, the constraint of distance between the pipe and the wall; Problem 2, the constraint of distance between pipes and the pipes relative location; Problem 3,the faint distance between pipes.

In order to solve above three problems. Two functions are proposed in this paper.

Function $S . S$ is to represent the state of the points in 3-dimensional environment. Every point's state is limited within $[0,1] . S$ (pi) $=1$ represents the point i is in obstacles, $S$ (pj) $=0$ represents the point $\mathrm{j}$ is free for design. Some pipes need lay out close to some points, so this kind of points' $S$ value should be lower, some pipes need lay out far away from some points, so this kind of points' $S$ value should be higher. In the process of individual evolution, the gene with lower state $S$ value could maintain to next generation until finally excellent individual satisfied with constraints appear.

Function $O . O$ is to represent the state of the points in 3-dimensional environment, which points can be affected by the relationship between pipes. In this paper, according to distance constraints between two pipes, when the first pipe has been designed, the second pipe are accordingly determined. The relation between these two pipes can represented by points. So relative points state should be recorded into $O$. Because only these two pipes exist the distance, that is pipe 1 influence 
pipe 2, not influence other pipes, $O$ must be changed according to different pipes distance constraints. After pipe 2 has been designed already, O's recorder should be clear to wait for the next pipe to lay out. In addition, during the period of waiting for the next pipe's design, $O$ equals $S$ for every point. The difference between $S$ and $O$ is $S$ records the global state of points, while $O$ records the dynamic state of points according to specific pipeline rules.

The evaluation method should first calculate the length of the pipeline, which can be done by counting the number of points in each pipeline. The number of points in each pipeline is different. Second, the evaluation method should calculate the bends' number. The judgment of a bend is based on the genetic code. Actually, for every three points' coordinate, comparing the first coordinate to the third coordinate. If two coordinate have two differences in $\mathrm{x}, \mathrm{y}$ or $\mathrm{z}$, then there exist a bend. Finally the evaluation method should evaluate the distance requirement referring to $S$ and $O$.

Based on analysis above, an evaluation method is given and the evaluation function is defined as Eq.1.

$$
F=1 /(\alpha L+\beta W+D+A) \text {, }
$$

Where $\mathrm{L}$ is the length of a pipeline, $\mathrm{N}$ is the number of points for a pipeline, therefore $\mathrm{L}=\mathrm{N}$. W is the number of bends for a pipeline. $\alpha, \beta$ are positive constants. The proposed evaluation method gives a penalty to infeasible paths, which paths are in obstacles when designing two relative pipelines, but still keeps them in the population pool because they might become good feasible solutions after certain genetic transformations. Importantly, this evaluation may allow some overlap between fitness of feasible and infeasible solutions by adjusting $D, E q$.2. It would be beneficial to give more chance to some good infeasible solution that would be easily to be evolved to good solutions.

$$
D(p)=\left\{\begin{array}{l}
1000 \\
0
\end{array}\right.
$$

If the second pipeline is in obstacles, $\mathrm{D}(\mathrm{p})=1000$; If the second pipeline is not in obstacles, $\mathrm{D}(\mathrm{p})=0$.

$$
A=\sum_{i=1}^{N} O i
$$

From $E q .3, A$ reflects the influence of the second pipeline to the first pipeline. $O i$ is the $O$ 's value for each point. $\mathrm{A}$ is desire to be lower.

\section{Genetic Operators}

Those three commonly-used basic genetic operators, select, crossover and mutation, are not applicable for proposed method. They have to be tailored to suit for the problem and the adopted problem representation.

Select is the operator that randomly choose some pipelines to the next generation. In order to keep the variety of chromosome, some modifications are made to the operator. Arrange the fitness of a generation first, then choose the chromosome from three parts, which generation's fitness are divided into three part from high fitness to low fitness. In each part, choose the chromosome

\begin{tabular}{|c|c|c|c|c|c|c|c|c|}
\hline $\mathrm{P} 1$ & $\mathrm{P} 2$ & $\mathrm{P} 3$ & $\mathrm{P} 4$ & P5 & P6 & P7 & P8 & P9 \\
\hline \multicolumn{9}{|c|}{ sort } \\
\hline P3 & $\mathrm{P} 5$ & $\mathrm{P} 2$ & P9 & $\mathrm{P} 4$ & $\mathrm{P} 7$ & $\mathrm{P} 1$ & P6 & $\mathrm{P} 8$ \\
\hline \multicolumn{9}{|c|}{ select } \\
\hline & & & P5 & P9 & P8 & & & \\
\hline
\end{tabular}
randomly.Fig.1.shows the process of select.

Parent 1

Fig.1.Select operator 
Crossover is the operator that randomly choose a node from Parent 1 and the other node from Parent 2.Exchange the part after these two points. However, the special genetic code in this paper could not use common crossover. As [5] mentioned, two kinds of crossover are suit for this special code.Mutation is the operator that randomly choose a node and replace it with a node that is not included in the path. Mutation is served as a key role to diversify the solution population. The mutation in this paper is different slightly. [5] choose two points randomly and produce a new pipeline with start point and target point using chosen two points. Aim to get feasible pipeline efficiently, based on [5], choose the lowest S's points, and four more points around this point. Then use new path to instead of this period of path with the same start point and target point.Fig.2.shows the process of mutation

Parent 1

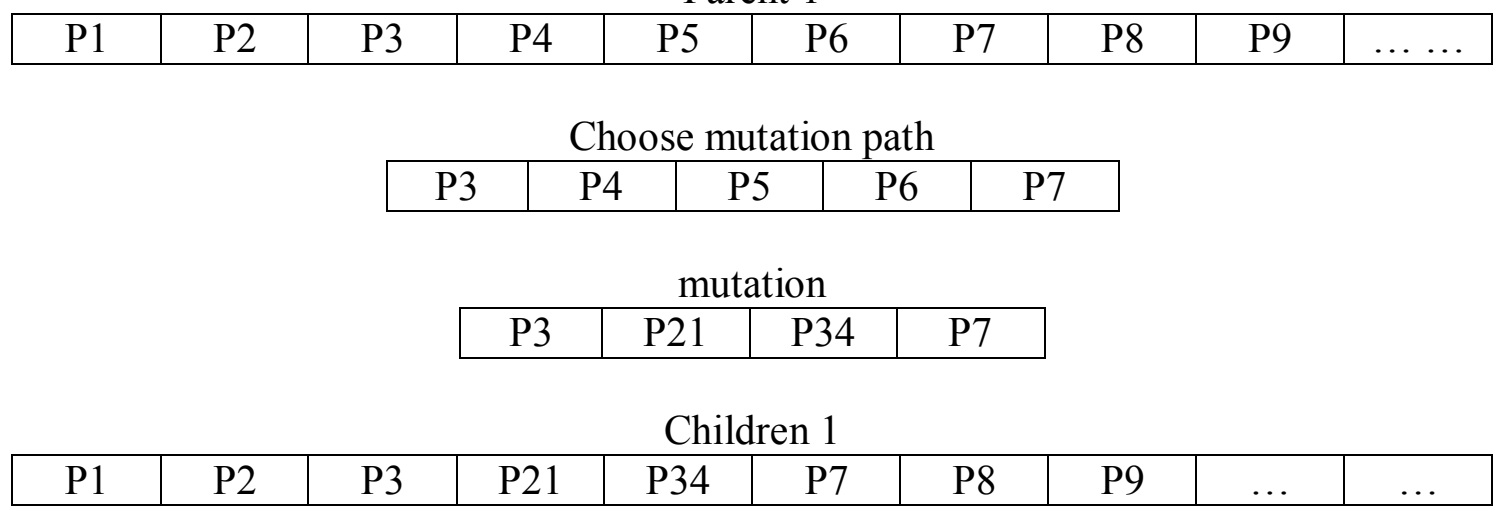

Fig.2.Mutation operator

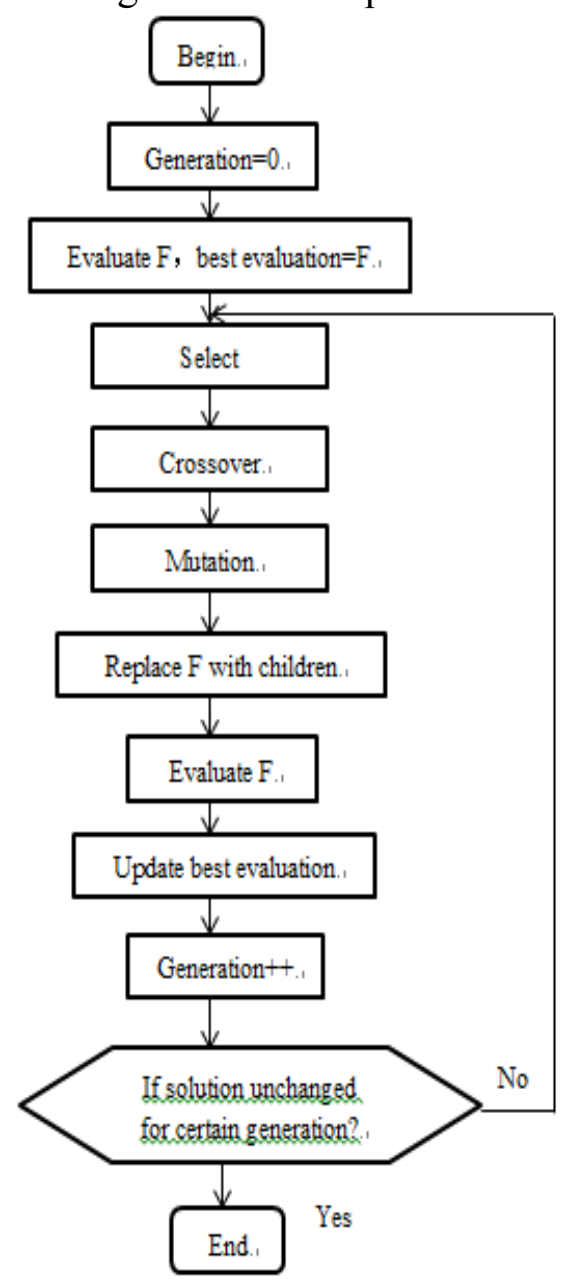

Fig.3. Outline of the method based genetic algorithm for pipe routing design. 


\section{Outline of the Method Based Genetic Algorithm}

An outline of the proposed method based genetic algorithm is given in Fig.3.Initial solution are generated randomly and evaluated by the fitness function in Eq.1.The population is evolved generation by generation. In each generation, genetic operators are applied to the whole population. After that, the population is replaced by the generated children with elitism. The use of elitism can prevent the GA from losing the good solutions found in the evolution and speed up convergence of the population. The best solution so far is updated in each generation, and it will be the final solution when some stop criterion is satisfied. The stop criterion can either be that the preset maximum generation is exceeded, or that the best solution remains unchanged for certain number of generations.

\section{Summary}

In this paper, a method based genetic algorithm for pipe routing design is proposed. The GA uses special path representation to represent environment. The classical genetic operators are tailored to the pipe routing design. The developed GA also features its fitness function to evaluate the pipe distance constraint. Two functions are added to help evaluate.

\section{References}

[1] Ito T. A genetic algorithm approach to piping route path planning[J]. Journal of Intelligent Manufacturing, 1999, 10(1):103-114.

[2] Xiaoning Fan, A study of optimization methods for ship pipe routing design and applications, Doctor's thesis, Dalian University of Technology, 2006.

[3] Xiaoning Fan, Yan Lin, Zhuoshang Ji. The Ant Colony Optimization for Ship Pipe Route Design in 3D Space. The sixth World Congress on Intelligent Control and Automization, 2006, 4:3103-3108.

[4]Bai Ming, Researches on Path Optimization Algorithm of Ship Pipe System, Master's thesis, Mechanical manufacture and Automation, Harbin Engineering University, 2010.

[5]Gao Yuefeng, Research on Engine Room Piping Automatic Layout Based on Genetic Algorithm , Master's thesis, Dalian Maritime University, 2014. 